\title{
Bacterial bloodstream infections in a tertiary infectious diseases hospital in Northern Vietnam: aetiology, drug resistance, and treatment outcome
}

Vu Quoc Dat ${ }^{1,2,3^{*}}$ D, Hieu Ngoc Vu' ${ }^{1}$, Hung Nguyen The ${ }^{1}$, Hoa Thi Nguyen ${ }^{1}$, Long Bao Hoang ${ }^{3}$, Dung Vu Tien Viet ${ }^{3}$, Chi Linh Bui ${ }^{1}$, Kinh Van Nguyen², Trung Vu Nguyen ${ }^{1,2}$, Dao Tuyet Trinh ${ }^{2}$, Alessandro Torre ${ }^{3}$, H. Rogier van Doorn³, Behzad Nadjm ${ }^{3,4}$ and Heiman F.L. Wertheim ${ }^{3,4,5}$

\begin{abstract}
Background: Bloodstream infections (BSIs) are associated with high morbidity and mortality worldwide. However their aetiology, antimicrobial susceptibilities and associated outcomes differ between developed and developing countries. Systematic data from Vietnam are scarce. Here we present aetiologic data on BSI in adults admitted to a large tertiary referral hospital for infectious diseases in Hanoi, Vietnam.

Methods: A retrospective study was conducted at the National Hospital for Tropical Diseases between January 2011 and December 2013. Cases of BSI were determined from records in the microbiology department. Case records were obtained where possible and clinical findings, treatment and outcome were recorded. BSI were classified as community acquired if the blood sample was drawn $\leq 48 \mathrm{~h}$ after hospitalization or hospital acquired if $>48 \mathrm{~h}$.
\end{abstract}

Results: A total of 738 patients with BSI were included for microbiological analysis. The predominant pathogens were: Klebsiella pneumoniae (17.5\%), Escherichia coli (17.3\%), Staphylococcus aureus (14.9\%), Stenotrophomonas maltophilia (9.6\%) and Streptococcus suis (7.6\%). The overall proportion of extended spectrum beta-lactamase (ESBL) production among Enterobacteriaceae was 25.1\% (67/267 isolates) and of methicillin-resistance in S. aureus (MRSA) 37\% (40/108). Clinical data was retrieved for 477 (64.6\%) patients; median age was 48 years (IQR 36-60) with 27.7\% female. The overall case fatality rate was $28.9 \%$ and the highest case fatality was associated with Enterobacteriaceae BSI (34.7\%) which accounted for $61.6 \%$ of all BSI fatalities.

Conclusions: Enterobacteriaceae (predominantly K. pneumoniae and E. coli) are the most common cause of both community and hospital acquired bloodstream infections in a tertiary referral clinic in northern Vietnam.

Keywords: bacteremia, bloodstream infection, Vietnam, sepsis, drug resistance, bacterial, Gram-negative bacteria, Streptococcus suis, Burkholderia pseudomallei

\footnotetext{
* Correspondence: datvq@hmu.edu.vn; quocdat181@yahoo.com

1Department of Infectious Diseases, Hanoi Medical University, Hanoi, Vietnam

${ }^{2}$ National Hospital for Tropical Diseases, Hanoi, Vietnam

Full list of author information is available at the end of the article
} 


\section{Background}

Bloodstream infections (BSIs) are an important cause of sepsis-related morbidity and mortality worldwide [1]. A systematic review of BSI burden in population based studies estimated the annual numbers of BSI episodes ranged from 575.462-677.389 in North America and $1,213,460-1,381,590$ in Europe with the annual numbers of BSI associated deaths were 79,466-93,655 and 57,750-276,318 respectively [2]. In developed setting, the BSI and BSI associated septic shock still accounted for $6 \%$ and $3 \%$ of all admission to ICUs with the inhospital mortality rates of $40 \%$ and $49 \%$ respectively [3]. Additionally, BSIs are associated with long-term risk of mortality among survivors [4]. In a population based study in Denmark, while the incidence of community and hospital acquired BSIs annually decreased 3.7\% and $4.2 \%$ respectively between 1998 to 2008, the incidence rate of healthcare-associated bloodstream infection BSI remained unchanged [4]. Data from low and middle income countries (LMIC) on the causes of bloodstream infection are limited [5], but crucial for enabling clinicians to direct antimicrobial therapy appropriately and for the design of preventive measures. Useful lessons can be learned from analysing the bacterial aetiology of BSIs as most cases reflect severe illness and bacteria detected are usually directly responsible for disease [6, 7]. The causative agent will be affected by a number of factors, particularly the focus of infection, comorbidities like immunodeficiency, chronic renal and liver disease, in addition to socio-economic, climatic and other geographical factors [8]. The level of economic development can also impact aetiology distribution through changing exposure to environmental organisms associated with urbanisation, changing farming and food consumption practices, vaccination and comorbidities associated with increased wealth and improved life expectancy [9]. Consequently, data from tropical emerging economies are important to understanding and updating worldwide trends of the aetiology of severe bacterial disease in the tropics.

Vietnam is a low middle income country (LMIC), an emerging economy and a hotspot for emerging infectious diseases [10]. Levels of antibiotic resistance, an important cause of treatment failure and subsequent mortality are also high in Vietnam [11, 12]. In the Asian Network for Surveillance of Resistant Pathogens with 11 participating countries in 2001, Vietnam had a highest prevalence of pneumococcal multidrug resistance (defined as resistance to at least three classes of antibiotics) (71.4\%) which much higher than the second and third highest countries and territories of Korea (54.8\%) and Hong Kong (43.2\%) [12]. In another study in 20 hospitals in 5 Asia-Pacific countries, the prevalence of carbapenem non-susceptible Gram-negative pathogens was highest at $35.0 \%$ in Vietnam, in which the prevalence of carbapenem non-susceptiblity among Pseudomonas aeruginosa, Enterobacteriaceae and Acinetobacter baumannii were $46.7 \%, 5.6 \%$ and $89.5 \%$ respectively [11].

In-hospital mortality among patients with sepsis is reduced by early initiation of adequate empiric antibiotic therapy $[13,14]$ and strategies to promote appropriate antibiotic use may help to prevent antimicrobial resistance and optimise use of hospital resources. However, the epidemiology of BSI in Vietnamese adults and associated treatment outcomes are not well studied. An understanding of BSI in Vietnam, its resistance patterns and their impact on patient's outcomes is important to guide clinical management and appropriate antibiotic use. This article reports on a retrospective analysis of three years of BSI data at the National Hospital for Tropical Diseases (NHTD), a large tertiary referral hospital for infectious diseases in Hanoi, Vietnam.

\section{Methods \\ Study design and data collection}

This is a retrospective, cohort study of patients with bacterial bloodstream infection (BSI) admitted to the National Hospital for Tropical Diseases (NHTD) between January 1st, 2011 and December 31st, 2013. NHTD is a 300-bed tertiary teaching hospital that provides care to individuals suspected of an infectious disease living within its catchment area. NHTD is also a referral hospital assigned to all provincial hospitals in the north of Vietnam for infectious diseases that cannot be managed locally. As a result the hospital receives patients both directly from the community and transferred from hospitals in the region. The study included all positive blood cultures with recognized bacterial pathogens among patients who were hospitalized during the study period and additional clinical information from a subgroup of patients whose patient records were available to the investigators. A standard data collection form was created to extract demographic \& clinical data, microbiology and outcome information from routine patient and microbiology records. Where patients had been transferred from another hospital, the transfer letter was examined for any record of antibiotic therapy having been provided at the referring hospital.

\section{Study definitions}

A recognised bacterial pathogen identified as an organism which is not on the CDC's National Healthcare Safety Network (NHSN) common commensal list unless there were 2 bottles from the same set were positive for the same organism or a single positive bottle plus an evident focus of infection (abscess or valvular vegetation). An episode of BSI was defined as the isolation of the 
organism(s) from one or more positive blood bottles which were explainable by a common source of infection and the time between two isolations was not interrupted by an asymptomatic period. When the clinical information was not available for judgment, the same organism(s) isolated within $48 \mathrm{~h}$ of another positive culture was considered as the same episode [15].

A case of community-acquired BSI (CABSI) was defined as a patient with a positive blood culture from a blood sample drawn $\leq 48 \mathrm{~h}$ after hospitalization at any health care facility (to ensure correct categorisation of patients referred from another hospital) for the current illness. Hospital-acquired BSI (HABSI) cases were defined as patients with $>48$ h between admission at any health care facility and the blood being drawn using the referring letter when applicable $[16,17]$. These data were only available from patients whose clinical records were available. Any BSI caused by Streptococcus suis or Burkholderia pseudomallei, was classified as CABSI regardless of when samples were taken with the assumption that the acquisition of those infection due to occupational exposure to pigs and environmental exposure to contaminated environment are unlikely in the hospital setting respectively $[18,19]$.

Blood for culture, approximately 5-10 mL of blood, was collected in a single commercially sourced aerobic bottle and incubated in an automated system (Bactec, Becton Dickinson, USA) for five days (5-10 mL blood). Bottles that flagged positive were Gram-stained, subcultured and identified using standard microbiological techniques, including VITEK and API testing (bioMérieux, France). Antibiotic susceptibilities were performed using CLSI guidelines with breakpoints from 2013 [20]. For S. suis, the susceptibility was evaluated using CLSI breakpoints for Streptococcus viridans group [21].

Fatal cases were defined as patients who died in hospital or were discharged with diagnosis of brain death and the expectation that they will die imminently within hours or days (palliative discharge to die at home, a common practice in Vietnam).

\section{Data entry and statistical analysis}

Data were entered in Epidata (EpiData Association, Odense, Denmark) and analysed using SPSS 22.0.0 (IBM Co., Armonk, NY, USA). Standard descriptive statistics were calculated for categorical (in percentage) and continuous variables (median and interquartile, IQR). Bivariate analyses were performed using Pearson's chi-squared test or Fisher's Exact Test for categorical variables as appropriate. The possible selection bias was assessed by chi-square and t-test analyses to compare gender and age differences between those with and without clinical outcomes, respectively. Cox proportional-hazards regression was used to identify variables that predict clinical outcomes. Cox's proportional hazards regression was performed to analyse factors associated with all-cause in-hospital mortality. BSI episodes of polymicrobial infection and S. maltophilia infections were excluded from regression analysis due to a small number and an uncertain pathogenicity respectively. In cases where there were multiple blood cultures positive, we only included the first positive blood culture. All tests were two-tailed and differences were considered statistically significant at $p$ values $\leq 0.05$.

\section{Results}

\section{Study population's demographics}

There were 894 positive blood cultures corresponding to 835 BSI episodes in 825 patients recorded between January 2011 and December 2013. Notes from 543/ $825(65.8 \%)$ patients could be retrieved to collect clinical details of individual patients while 282 (34.2\%) were not available (Fig. 1). Sensitivity analysis of age and gender showed no differences between cases with and without clinical outcome (chi-square test, $p=0.664$ and t-test, $p=0.053$ respectively). Among collected records, sixty-six cases $(12.2 \%, 66 / 543)$ were subsequently classified by cultured organism as having a pseudo-bacteremia due to contamination, including 16 cases with Burkholderia cepacia, 19 cases with Alcaligenes species, 5 cases with Ralstonia pickettii and 4 cases with Pseudomonas species (2 P. putida and $2 P$. stutzeri). There were 3 cases with Serratia marcescens, 3 S. maltophilia, $1 \mathrm{~K}$. pneumoniae and 1 $E$. coli which were classified as contaminants by treating doctors at the time of results return, based on patient's survival and recovery without antibiotic treatment for bacterial disease and with an alternative possible viral diagnosis. Other contaminated cultures were 3 cases with Ochrobactrum anthropi, 1 case with Chryseobacterium indologenes, 1 case with Chryseomonas luteola, 1 case with (non-diphtheroid) Corynebacterium spp., 1 case with Staphylococcus spp. and 7 cases with single culture of viridans group streptococci without an endovascular or other focus. As a result, 477 cases were included for the clinical analysis.

The median age of the group of 477 patients with BSI was 48 years (IQR 36-60), 27.7\% of patients were female (132/477), 14 (10.6\%) of whom were pregnant). Common co-morbidities were chronic hepatitis or cirrhosis $(14.3 \%, 68 / 477)$, diabetes mellitus $(7.1 \%, 34 / 477)$ and HIV infection $(6.3 \%, 30 / 477)$ (Table 1$)$.

In patients with CABSIs, $12.5 \%$ (50/400) had multiple positive blood cultures with either $S$. aureus or K. pneumoniae. Whilst most patients with CABSI were admitted directly from the community, 153/400 patients $(38.3 \%)$ were transferred from other hospitals within $48 \mathrm{~h}$, with $62 / 153$ patients $(40.5 \%)$ recorded as 


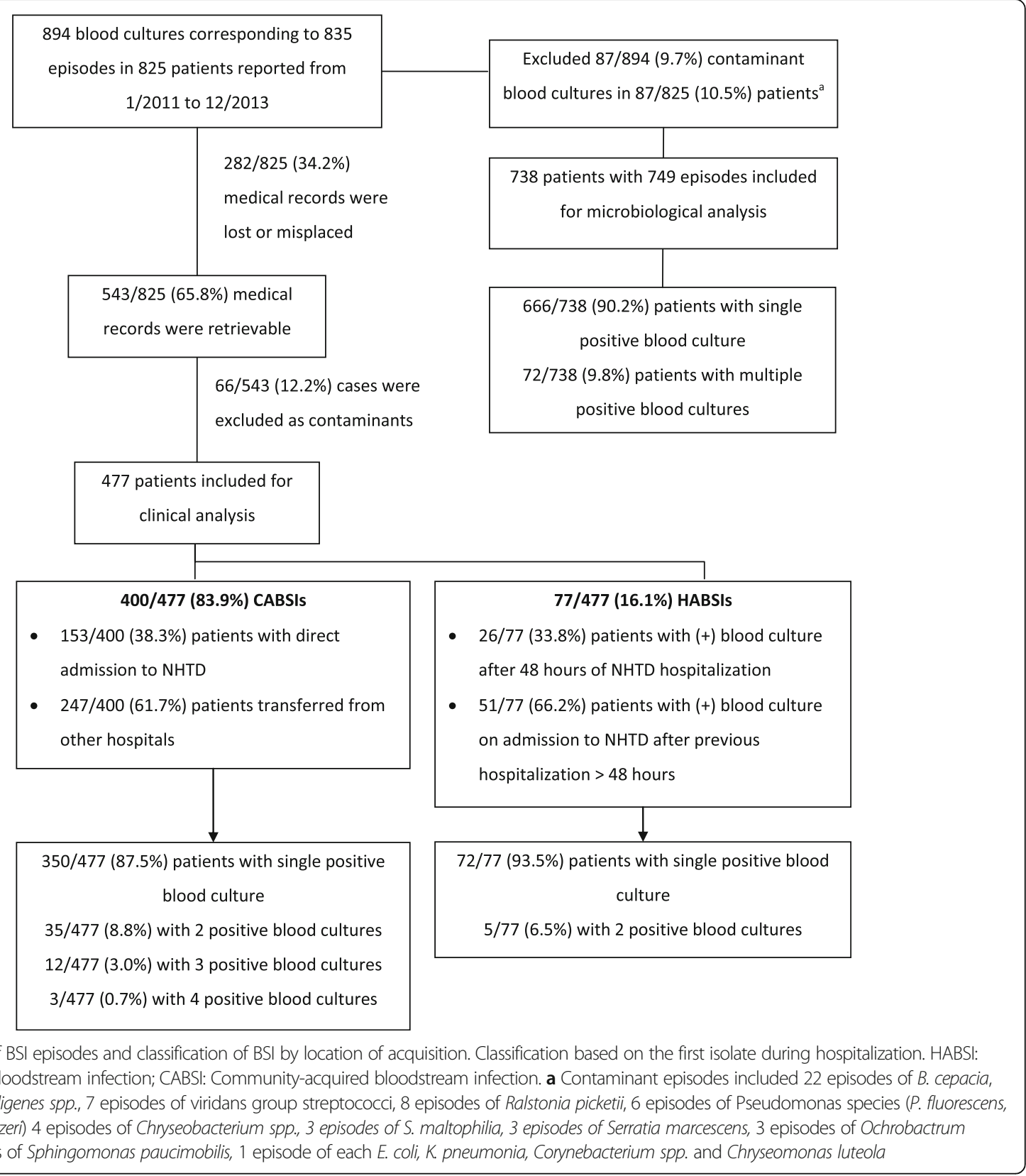

having received antibiotics before admission to the study hospital.

\section{Aetiology of bloodstream infections Analysis of microbiological dataset}

During the 3 year period, a total of 738 BSI patients were identified with $67.9 \%$ (501/738) with Gramnegative bacteria isolated, 31\% (229/738) Gram-positive bacteria, and $1.1 \%(8 / 738)$ had two pathogens isolated in the same culture bottle $(n=8)$. Enterobacteriaceae were the most frequently isolated group of organisms among patients with BSI $(46.7 \%, 345 / 738)$ with the predominant Enterobacteriaceae being K. pneumoniae (37.4\%, 129/
345) and E. coli $(37.1 \%, 128 / 345)$, followed by Salmonella enterica ( $13.0 \%$ or $45 / 345)$ (Table 2 ).

Among Gram-positive isolates, S. aureus was the leading pathogen $(48 \%$ or $110 / 229)$, followed by S. suis $(24.5 \%$ or 56/229) and other Streptococcus species (16.2\% or 37/229). Non- Enterobacteriaceae Gram-negative bacteria were identified in 156 cases (21.1\%), of which S. maltophilia accounted for $45.5 \%$ (71/156).

The overall proportion of extended spectrum betalactamases (ESBL) production among Enterobacteriaceae was $25.1 \%$ (67/267 isolates, 78 missing result). Tables 3 and 4 display the antibiotic resistance of $K$. pneumoniae and E. coli. The prevalence of ESBL production among 
Table 1 Characteristics of the clinical study population

\begin{tabular}{llll}
\hline & Total & Community acquired BSI & Hospital acquired BSI \\
$N=77$ & $N=400$ & $n(\%)$ & $n(\%)$ \\
& $N=477$ & $48(36-60)$ & $50(32-60)$ \\
\hline Age - median (IQR) & $48(36-60)$ & $122(30.6 \%)$ & $10(13 \%)$ \\
Female sex & $132(27.7 \%)$ & $190(47.5 \%)$ & $55(71.4 \%)$ \\
Any ICU admission during NHTD hospitalisation & $245(51.4 \%)$ & $109(27.3 \%)$ & $33(42.9 \%)$ \\
Any history of medical condition & $142(29.8 \%)$ & $54(13.5 \%)$ & $14(18.2)$ \\
Liver disease & $68(14.3 \%)$ & $25(6.3 \%)$ & $9(11.7 \%)$ \\
Diabetes & $34(7.1 \%)$ & $6(1.5 \%)$ & $3(3.9 \%)$ \\
Moderate or severe renal disease & $9(1.9 \%)$ & $21(5.3 \%)$ & $9(11.7 \%)$ \\
HIV infection & $30(6.3 \%)$ & $8(2 \%)$ & $2(2.6 \%)$ \\
Other morbidity & $10(2.1 \%)$ & $7(1.8 \%)$ & $1(1.3 \%)$ \\
Long-term corticosteroid use & $8(1.7 \%)$ & $56(14 \%)$ & $11(14.3 \%)$ \\
Self-report alcoholism & $67(14 \%)$ & $17(4.3 \%)$ & $6(7.8 \%)$ \\
Intravenous drug use & $23(4.8 \%)$ & & \\
\hline
\end{tabular}

Liver disease was defined in a patient with chronic hepatitis and/or cirrhosis

${ }^{\mathrm{b}}$ Moderate or severe renal disease was defined in a patient with baseline creatinine $>3 \mathrm{mg} \%$ ( 265 umol/l), dialysis or kidney transplantation

E. coli isolates was significantly higher than in $K$. pneumoniae (45\%, 50/111 vs 12.3\%,15/122, $p<0.001$ ). Among Gram-positive bacteria, 97.4\% (38/39) of S. suis isolates were susceptible to penicillin with penicillin MIC median of 0.032 (IQR $0.023-0.032$ ) and only 1 case was intermediate susceptible to penicillin with an MIC of 0.23 , though no confirmatory testing was performed. Methicillin resistance was observed in 37\% (40/108) of $S$. aureus isolates and all 29 MRSA isolates tested with vancomycin E-test were susceptible. Among $67 \mathrm{~S}$. aureus isolates tested for vancomycin by E-test, 62.7\% (42/67) of isolates had vancomycin MIC $\leq 1 \mu \mathrm{g} / \mathrm{ml}$ and $37.3 \%$ (25/67) 1.5-2 $\mu \mathrm{g} / \mathrm{ml}$ (Table 5).

\section{Analysis of the clinical dataset}

The proportions of CABSI and HABSI caused by Enterobacteriaceae were not statistically different (55.1\%, 203/397 vs 58.4\%, 45/77, $p=0.24)$, similar for for non-enterobacteriaceae Gram-negative bacteria (19.6\%, 78/397 vs $27.3 \%, 21 / 77, p=0.132)$. In contrast, Gram-positive bacteria were more frequently identified in CABSI (29.2\%, 116/397 vs $14.3 \%, 11 / 77$, $p=0.011)$ and Acinetobacter species were more common in HABSI (Table 2).

There was no difference in the proportion of ESBL production in community versus hospital setting among E. coli isolates $(53.8 \%, 28 / 52$ vs $55.6 \%, 5 / 9, p=1.0)$. In K. pneumoniae isolates, the proportion of ESBL production were higher in HABSI $(21.1 \%, 4 / 19$ vs $6.1 \%, 5 / 82$ vs, $p=0.039)$. Compared with $K$. pneumoniae, E. coli isolates were also more frequently resistant to aminoglycosides $(23.3 \%, 30 / 129$ vs $13.0 \%, 17 / 131, p=0.031)$ and fluoroquinolones $(31.8 \%, 41 / 128$, vs $8.4 \%, 11 / 131$, $p<0.001)$.

K. pneumoniae isolates associated with HABSI more frequently showed antibiotic resistance compared to CABSI isolates, though statistical significance was only reached for quinolones, while the pattern of resistance among $E$. coli isolates did not differ between the hospital and community (Tables 3 and 4 ). MRSA rates differed between community $(38.7 \%, 12 / 31)$ and hospital settings $(0 / 7)$, though this did not reach statistical significance $(p=0.074)$. Case notes were not retrievable in $69 / 110$ (62.7\%) cases of $S$. aureus bacteremia.

\section{Case fatality of bloodstream infection}

Case fatality was assessed in the clinical dataset only. The overall in-hospital case fatality rate among patients was $138 / 477$ (28.9\%). Overall case fatality rates among patients infected with Enterobacteriaceae, non-Enterobacteriaceae and Gram-positive bacteria were 34.7\% (85/245), 29.3\% (29/99) and 19\% (24/126), respectively (Fig. 2). There was no case of death among patients with mix infections (0/7). Case fatality rate in CABSI $(27.5 \%, 110 / 400)$ was lower, but not statistically different from HABSI (36.4\%, 28/77) $(p=0.131)$. In the most frequent isolates of $E$. coli, K. pneumoniae, S. aureus, S. maltophilia and S. suis, case-fatality rates were $35.6 \%(26 / 73), 35.5 \%$ (39/110), $26.8 \%(11 / 41), 17.0 \%(8 / 39)$ and $9.3 \%(4 / 39)$.

Multivariate Cox proportional hazard regression analysis was used to measure the effect of demographics, acquisition of infection, co-morbidities, previous hospital referring factors and bacterial species. Independent factors associated with case fatality were Enterobacteriaceae 
Table 2 Aetiology of bloodstream infections

\begin{tabular}{|c|c|c|c|c|c|}
\hline \multirow[t]{3}{*}{ Pathogen } & \multirow{3}{*}{$\begin{array}{l}\text { All patients } \\
N=738\end{array}$} & \multicolumn{4}{|c|}{ Sampled clinical cases } \\
\hline & & \multirow{2}{*}{$\begin{array}{l}\text { Total } \\
N=477\end{array}$} & \multirow{2}{*}{$\begin{array}{l}\text { CABSI } \\
N=400\end{array}$} & \multirow{2}{*}{$\begin{array}{l}\text { HABSI } \\
N=77\end{array}$} & \multirow[t]{2}{*}{$p^{9}$} \\
\hline & & & & & \\
\hline Enterobacteriaceae & $345(46.7 \%)$ & $245(51.4 \%)$ & $200(50 \%)$ & $45(58.4 \%)$ & 0.213 \\
\hline Klebsiella pneumoniae & $129(17.5 \%)$ & $110(23.1 \%)$ & $88(22 \%)$ & $22(28.6 \%)$ & $0.237^{d}$ \\
\hline Escherichia coli & $128(17.3 \%)$ & $73(15.3 \%)$ & $64(16 \%)$ & $9(11.7 \%)$ & 0.391 \\
\hline Salmonella enterica & $45(6.1 \%)$ & $30(6.3 \%)$ & $24(6 \%)$ & $6(7.8 \%)$ & 0.606 \\
\hline serovar Typhi & $10(1.4 \%)$ & $4(0.8 \%)$ & $4(1.00 \%)$ & 0 & 1 \\
\hline non-typhi Salmonella & $35(4.7 \%)$ & $26(5.5 \%)$ & $20(5 \%)$ & $6(7.8 \%)$ & 0.407 \\
\hline Serratia marcescens & $16(2.2 \%)$ & $12(2.5 \%)$ & $9(2.3 \%)$ & $3(3.9 \%)$ & 0.421 \\
\hline Enterobacter species & $16(2.2 \%)$ & $12(2.5 \%)$ & $8(2.0 \%)$ & $4(5.2 \%)$ & 0.112 \\
\hline Other Enterobacteriaceae species ${ }^{a}$ & $11(1.5 \%)$ & $8(1.7 \%)$ & $7(1.8 \%)$ & $1(1.3 \%)$ & 1 \\
\hline Non-Enterobacteriacae & $156(21.1 \%)$ & 99 (20.8\%) & $78(19.5 \%)$ & $21(27.3 \%)$ & 0.127 \\
\hline Stenotrophomonas maltophilia & $71(9.6 \%)$ & $47(9.9 \%)$ & $39(9.8 \%)$ & $8(10.4 \%)$ & 0.836 \\
\hline Aeromonas species & $34(4.6 \%)$ & $14(2.9 \%)$ & $12(3.0 \%)$ & $2(2.6 \%)$ & 1 \\
\hline Burkholderia pseudomallei & $15(2.0 \%)$ & $13(2.7 \%)$ & $13(3.3 \%)$ & 0 & \\
\hline Pseudomonas aeruginosa & $16(2.2 \%)$ & $13(2.7 \%)$ & $9(2.3 \%)$ & $4(5.2 \%)$ & 0.240 \\
\hline Acinetobacter species ${ }^{\mathrm{b}}$ & $16(2.2 \%)$ & $10(2.1 \%)$ & $3(0.8 \%)$ & $7(9.1 \%)$ & $<0.001$ \\
\hline Other gram-negative bacteriac & $4(0.5 \%)$ & $2(0.4 \%)$ & $2(0.5 \%)$ & 0 & 1 \\
\hline Gram-positive isolates & $229(31.0 \%)$ & $126(26.4 \%)$ & $115(28.8 \%)$ & $11(14.3 \%)$ & 0.007 \\
\hline Staphylococcus aureus & $110(14.9 \%)$ & $41(8.6 \%)$ & $34(8.5 \%)$ & $7(9.1 \%)$ & 0.826 \\
\hline Streptococcus suis & $56(7.6 \%)$ & $43(9.0 \%)$ & $43(10.8 \%)$ & 0 & - \\
\hline Streptococcus species & $37(4.9 \%)$ & $23(4.8 \%)$ & $21(5.3 \%)$ & $2(2.6 \%)$ & 0.559 \\
\hline S. pneumoniae & $7(0.9 \%)$ & $5(1.0 \%)$ & $4(1.0 \%)$ & $1(1.3 \%)$ & 0.587 \\
\hline S. viridans group ${ }^{d}$ & $21(2.8 \%)$ & $11(2.3 \%)^{b}$ & $10(2.5 \%)$ & $1(1.3 \%)$ & 1 \\
\hline Beta-hemolytic streptococci & $9(1.2 \%)$ & $7(1.5 \%)$ & $7(1.8 \%)$ & 0 & 0.604 \\
\hline Enterococcus species & $14(1.9 \%)$ & $12(2.5 \%)$ & $10(2.5 \%)$ & $2(2.6 \%)$ & 1 \\
\hline Other gram-positive bacteria & $12(1.6 \%)$ & $7(1.5 \%)$ & $7(1.8 \%)$ & 0 & 0.604 \\
\hline Polymicrobial $^{f}$ & $8(1.1 \%)$ & $7(1.5 \%)$ & $7(1.8 \%)$ & 0 & 0.604 \\
\hline
\end{tabular}

Including 3 cases with Klebsiella oxytoca, 3 cases with Proteus mirabilis, 2 cases with Escherichia hermannii, 1 case with Citrobacter freundii, 1 case with Morganella morganii and 1 case with Vibrio vulnificus

${ }^{\mathrm{b}}$ Including 12 cases with A. baumannii, 3 cases with A. Iwoffii and 1 case with A. junii

Including 1 case for Elizabethkingia meningosepticum, Haemophilus influenzae, Pasteurella multocida and Neisseria meningitidis,

${ }^{d}$ Cases with viridans group infection had at least two positive blood culture or single positive blood culture plus vegetation on echocardiography

e Including 4 cases with Rhodococcus equi, 3 cases with Listeria monocytogenes, 2 cases with Aerococcus viridians, 2 cases with Listeria spp. and 1 case with Listeria innocua

IIn 8 dual infection cases, 3 HIV infected cases had co-infection with Talaromyces marneffei and S. aureus, Escherichia hermannii or Salmonella group D; 1 case with S. aureus and K. pneumoniae, 1 case with Enterococcus faecalis and viridans streptococci, 1 case with $E$. coli and $K$. pneumoniae and 1 case with $S$. aureus and $E$. coli co-infection

${ }^{g} p$ values were calculated using Chi-square test or Fisher's Exact test as appropriate

and non-Enterobacteriaceae infection (HR 2.485, 95\% CI 1.546-3.994 and HR 1.931, 95\% CI 1.063-3.50, respectively), co-morbidities (HR 1.467, 95\% CI 1.017-2.114) and referring from other hospital (HR 2.049, 95\% CI 1.411-2.975) (Table 6).

\section{Discussion}

This retrospective study includes 3-years of complete microbiological data on aetiology of BSI for 738 patients and almost two thirds $(64.6 \%, 477 / 738)$ of these patients for clinical outcome analysis in a tertiary hospital in the north of Vietnam.

Our study shows the important role of pathogenic Gram-negative bacteria with a high proportion of cephalosporin-resistant E. coli and K. pneumoniae isolates and high prevalence of MRSA in CABSI. Our study shows the high contribution of Enterobacteriaceae BSI to mortality among adults admitted to our hospital in Vietnam, with over $60 \%$ of deaths associated with this group of bacteria. This study supports the observed shift 
Table 3 Resistance among K. pneumoniae from BSI

\begin{tabular}{|c|c|c|c|c|c|}
\hline \multirow[t]{2}{*}{ Resistance } & \multirow[t]{2}{*}{ All isolates } & \multicolumn{4}{|c|}{ Sampled clinical cases } \\
\hline & & Total & CABSI & HABSI & $P$ value $^{a}$ \\
\hline Any extended spectrum cephalosporin & 19/133 (14.3\%) & $13 / 112(11.6 \%)$ & $8 / 90(8.9 \%)$ & $5 / 22(22.7 \%)$ & 0.128 \\
\hline ESBL production & 15/122 (12.3\%) & 9/101 (8.9\%) & $5 / 82(6.1 \%)$ & $4 / 19(21.1 \%)$ & 0.062 \\
\hline Others & 4/133 (3.0\%) & $4 / 112(3.6 \%)$ & $3 / 90(3.3 \%)$ & $1 / 22(4.5 \%)$ & 1 \\
\hline Any carbapenem & 1/131 (0.8\%) & $1 / 109(0.9 \%)$ & 0/89 (0\%) & $1 / 20(5 \%)$ & 0.183 \\
\hline Any aminoglycoside & 17/131 (13.0\%) & 13/109 (11.9\%) & $9 / 89(10.1 \%)$ & $4 / 20(20 \%)$ & 0.252 \\
\hline Any fluoroquinolone & $11 / 131(8.4 \%)$ & $8 / 109(7.3 \%)$ & $4 / 89(4.5 \%)$ & $4 / 20(20 \%)$ & 0.036 \\
\hline Co-trimoxazole & 24/124 (19.4\%) & 19/102 (18.6\%) & $14 / 84(16.7 \%)$ & $5 / 18(27.8 \%)$ & 0.318 \\
\hline
\end{tabular}

${ }^{\mathrm{a}} p$ values were calculated using Fisher's Exact Test

within Enterobacteriaceae organisms from typhoidal and non-typhoidal Salmonella to K. pneumoniae and E. coli, the current leading causes of community acquired blood stream infections.

In Vietnam, Salmonella enterica serovar Typhi was reported to be the major cause of BSI, accounting for up to $74 \%$ of isolates from blood in the 1990 s but gradually declined to $6 \%$ by 2008 [22]. With the recent emergence of $K$. pneumoniae and E.coli, we observed the high prevalence of extended spectrum Beta-lactamase (ESBL) production in these pathogens, especially in the hospital setting. In surveillance data from five Asia-Pacific countries in 2010, Vietnam reported the highest rate of ESBL positive Enterobacteriaceae isolates with an overall prevalence of $55.1 \%$ and $80.1 \%$ in ICU patients [11]. The high prevalence of antibiotic resistance in Vietnam may be attributed to multiple factors, such as dispensing practices in the community, lack of enforcement of regulation, limited resources for microbiological diagnostics, injudicious use of antibiotics in hospital, community and agriculture and insufficient antibiotic resistance surveillance and infection control [23].

In our study, ESBL production was detected among $6.1 \% \mathrm{~K}$. pneumoniae from CABSI, which is consistent with the low ESBL prevalence of $4.1 \%$ among $K$. pneumoniae oropharyngeal carriers reported previously from northern Vietnam [24]. The rate of ESBL-production in E. coli(45\%) was higher than in Thailand (CABSI, $11.8 \%$ ) and Korea (31.3\% in HABSI and $8.8 \%$ in CABSI) and comparable to the prevalence of ESBL among E. coli isolates in BSI in Cambodia (47.7\%), [15, 17, 25]. The high rate of ESBL carriage in E. coli from CABSI is deeply worrying and likely reflects extensive overuse of oral cephalosporins in the community, their use in agriculture or other environmental sources [26, 27]. S. suis is a wellrecognized zoonotic pathogen associated with bacterial meningitis and BSI among adults in Vietnam [10] and was noted as an important Gram-positive pathogen cultured from blood with high levels of susceptibility to penicillin.

Our study showed a higher case-fatality rate $(110 / 400$ or $27.5 \%$ ) among community-acquired bacterial bloodstream infections than the rate of $9 \%$ reported in a review from 1990 to 2010 in south and southeast Asia and the rate of $18.1 \%$ among patients with CABSI in a meta-analysis in Africa from 1984 to 2006 [28, 29]. This difference is likely related to the changes in spectrum of aetiology with higher prevalence of Salmonella spp. (53.2\% of Enterobacteriaceae isolates) in the past [28], the emergence of high levels of antibiotic resistance in Vietnam and the tertiary referral nature of the study hospital. The overall mortality in our study was slightly higher than the overall mortality of $22.5 \%$ in Cambodia

Table 4 Resistance among E. coli from BSI

\begin{tabular}{|c|c|c|c|c|c|}
\hline \multirow[t]{2}{*}{ Resistance } & \multirow[t]{2}{*}{ All isolates } & \multicolumn{4}{|c|}{ Sampled clinical cases } \\
\hline & & Total & CABSI & HABSI & $P$ value $^{a}$ \\
\hline Any extended spectrum cephalosporin & $72 / 131(55.0 \%)$ & $45 / 74(60.8 \%)$ & $39 / 65(60 \%)$ & $6 / 9(66.7 \%)$ & 1 \\
\hline ESBL production & $50 / 111(45 \%)$ & $33 / 61(54.1 \%)$ & $28 / 52(53.8 \%)$ & $5 / 9(55.6 \%)$ & 1 \\
\hline Others & 22/131 (16.8\%) & $12 / 74(16.2 \%)$ & $11 / 65(16.9 \%)$ & $1 / 9(11.1 \%)$ & 1 \\
\hline Any carbapenem & $1 / 130(0.8 \%)$ & $0 / 73(0.0 \%)$ & $0 / 64(0.0 \%)$ & $0 / 9(0.0 \%)$ & - \\
\hline Any aminoglycoside & $30 / 129(23.3 \%)$ & $15 / 73(20.5 \%)$ & $14 / 64(21.9 \%)$ & $1 / 9(11.1 \%)$ & 0.675 \\
\hline Any fluoroquinolone & $41 / 129$ (31.8\%) & $26 / 72(36.1 \%)$ & $22 / 63(34.9 \%)$ & $4 / 9(44.4 \%)$ & 0.714 \\
\hline Cotrimoxazole & $62 / 90(68.9 \%)$ & $27 / 38(71.1 \%)$ & 24/33 (72.7\%) & $3 / 5(60.0 \%)$ & 0.615 \\
\hline Fosfomycin & $1 / 52(1.9 \%)$ & $1 / 44(2.3 \%)$ & $1 / 38(2.6 \%)$ & $0 / 6(0 \%)$ & - \\
\hline
\end{tabular}

${ }^{a} p$ values were calculated using Fisher's Exact Test 
Table 5 Resistance among S. aureus from BSI

\begin{tabular}{|c|c|c|c|c|c|}
\hline \multirow[t]{2}{*}{ Susceptibility } & \multirow[t]{2}{*}{ All isolates } & \multicolumn{4}{|c|}{ Sampled clinical cases } \\
\hline & & Total & CABSI & HABSI & $P$ value $^{a}$ \\
\hline Methicillin (MRSA) & $40 / 108$ (37\%) & $12 / 38(31.6 \%)$ & $12 / 31(38.7 \%)$ & $0 / 7(0 \%)$ & 0.074 \\
\hline Erythromycin & $39 / 72(54.2 \%)$ & 11/23 (47.8\%) & 10/18 (55.6\%) & $1 / 5(20 \%)$ & 0.317 \\
\hline Clindamycin & $56 / 109$ (51.4\%) & $21 / 40(52.5 \%)$ & 18/23 (54.5\%) & $3 / 7(42.9 \%)$ & 0.689 \\
\hline Chloramphenicol & 35/105 (33.3\%) & 14/38 (36.8\%) & $11 / 31(35.5 \%)$ & $3 / 7(42.9 \%)$ & 1 \\
\hline Gentamicin & 18/76 (23.7\%) & 9/26 (34.6\%) & $7 / 21$ (33.3\%) & $2 / 5(40 \%)$ & 1 \\
\hline Ciprofloxacin & 19/99 (19.2\%) & 7/33 (21.2\%) & 6/28 (21.4\%) & $1 / 5(20 \%)$ & 1 \\
\hline Levofloxacin & 18/103 (17.5\%) & 9/40 (22.5\%) & 6/13 (18.2\%) & $3 / 7$ (42.9\%) & 0.316 \\
\hline Co-trimoxazole & $8 / 62$ (12.9\%) & $5 / 28(17.9 \%)$ & 4/21 (19\%) & 1/7 (14.3\%) & 1 \\
\hline Rifampicin & 10/95 (10.5\%) & 6/33 (18.2\%) & $5 / 27(18.5 \%)$ & $1 / 6(16.7 \%)$ & 1 \\
\hline Vancomycin ${ }^{b}$ & 0/63 (0\%) & - & - & - & - \\
\hline
\end{tabular}

${ }^{a} p$ values were calculated using Fisher's Exact Test

${ }^{\mathrm{b}}$ The vancomycin susceptibility and the MRSA rate were defined by Minimum Inhibitory Concentrations (MICs) using E-test

where the epidemiology and pattern of resistance are similar [15]. We reported a high mortality of 35.6\% among E. coli BSI, which is higher than mortality of $18.2 \%$ and $8 \%$ and in a large surveillance in England and in a population-based study in Finland [30,31]. It is not clear about the reasons of the differences, but it may relate to pneumonia focus, severity of infection, antibiotic susceptibilities and seasonal variation [31]. As NHTD is a tertiary referral hospital, potentially more severe cases or cases that have failed therapy elsewhere may have been referred as compared to the hospitals included in other studies, leading to higher resistance and case fatality rates.

In North America and Europe, the nosocomial BSI case fatality rate ranges from 12 to $32 \%$ [2]. Changes in

Table 6 Cox proportional hazards model of factors associated with all-cause in-hospital case fatality among patients with bloodstream infections due to a single organism in clinical dataset

\begin{tabular}{|c|c|c|}
\hline Variable & $\begin{array}{l}\text { Hazard ratio } \\
(95 \% \mathrm{Cl})^{\mathrm{a}}\end{array}$ & $P$-value \\
\hline Gender (male/female) & $1.347(.857-2.115)$ & 0.196 \\
\hline Age (1-yr. increment) & $1.008(0.998-1.01)$ & 0.118 \\
\hline \multicolumn{3}{|l|}{$\begin{array}{l}\text { Aetiology of BSI (gram-positive } \\
\text { bacterial infection as reference) }\end{array}$} \\
\hline Enterobacteriaceae infections & $2.485(1.546-3.994)$ & $<0.001$ \\
\hline $\begin{array}{l}\text { Non Enterobacteriaceae } \\
\text { Gram-negative infections }\end{array}$ & $1.931(1.063-3.50)$ & 0.031 \\
\hline $\begin{array}{l}\text { Acquisition of infection } \\
\text { (CABSI as reference) }\end{array}$ & $1.006(0.633-0.399)$ & 0.053 \\
\hline Any co-morbidities & $1.467(1.017-2.114)$ & 0.040 \\
\hline Referring from another hospital & $2.049(1.411-2.975)$ & $<0.001$ \\
\hline
\end{tabular}

${ }^{a}$ Cases with S. maltophilia infection were excluded from analysis

bHazard ratios and $P$-values were calculated using Cox proportional hazards model the aetiology of HABSI began in the 1960s-1970s in the United States of America (US) with replacement of Gram-negative by Gram-positive organisms, such that $65 \%$ of BSIs were $S$. aureus, the most common nosocomial pathogen [32]. However, a Vietnamese population based study indicated $S$. aureus nasopharyngeal carriage proportion (33.8\%, CI 29.4-38.8) was higher than in European regions and the States [33], and S. aureus BSI is still mainly community-acquired in Vietnam [34]. In Vietnam the proportion of BSI attributable to $S$. aureus did not change during the 15-years from 1994 to 2008 [22]. Gram-negative bacilli are still recognized as the most frequent nosocomial isolates in developing countries [35]. In a multicenter surveillance study in Thailand, Gram-negative bacteria accounted for $67.6 \%$ of HABSI, of which Acinetobacter spp. (16.2\%) was detected most frequently, followed by $K$. pneumoniae (13.9\%) and S. aureus (13.9\%) and the in-hospital mortality was $26.6 \%$ [36]. The overall case fatality rate of $36.4 \%$ among HABSI in our study population was higher than seen in North America and Europe. This may be related to higher levels of antibiotic resistance, differences in the availability of resources or the nature of admissions to NHTD, as described earlier.

We observed a predominance of males among patients with BSI (72.3\%). This phenomenon was similar as in the cause of death reports in another tertiary hospital in the north of Vietnam where the top three of causes of death were septic shock, intracerebral haemorrhage and pneumonia. The proportion of males in this report was higher than females (1577/2340 or 67.4\%) [37].

Among common isolates causing BSI, we reported the high prevalence of S. maltophilia $(9.6 \% .71 / 738)$ which is only rarely considered a pathogen, particularly from the community [32]. Whilst $S$. maltophilia was described as a recognised cause of CABSI among patients with 


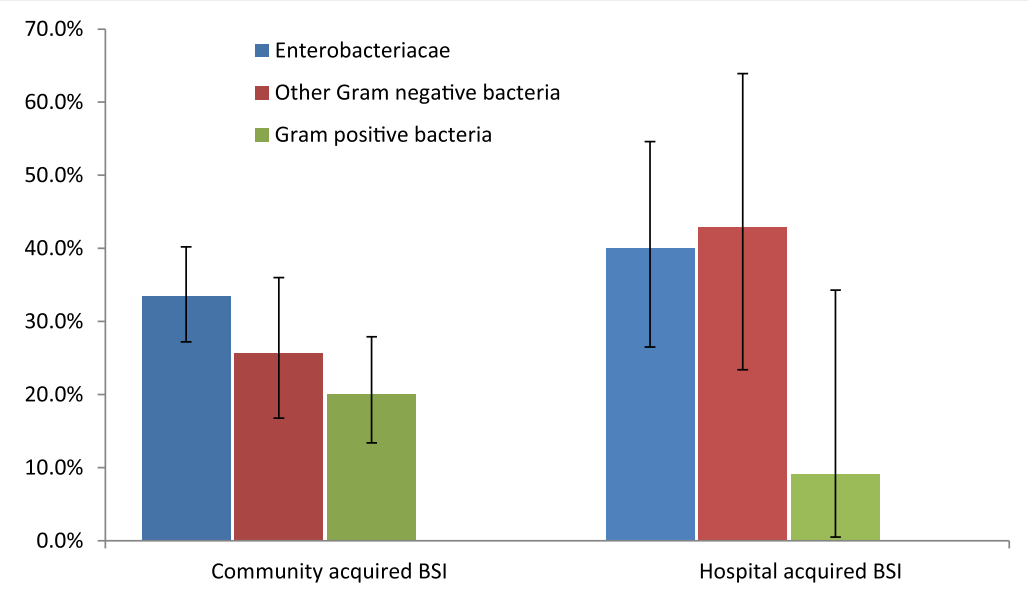

Fig. 2 Case-fatality by etiology of bloodstream infection (BSI). Error bars represent the $95 \%$ confidence interval (Cl) for case mortality proportions

comorbidities in a systematic review [38] and a recent publication from Taiwan [39], another publication describes contamination of blood culture bottles with $S$. maltophilia due to growth of the bacteria in disinfectant [40]. Furthermore, we have observed pseudoepidemics of S. maltophilia in this hospital (multiple patients from geographically disparate sites with different infection syndromes, newly admitted on the same ward with $S$. maltophilia cultured from blood at the same time). The retrospective nature of this study makes it difficult to define whether these isolates were true pathogens or represented contamination. Given how frequently this organism was isolated, further prospective investigation is required.

Our study has some important limitations. Firstly, NHTD is a tertiary referral hospital and specializes in infectious diseases. Therefore, the pattern of aetiology, resistance and spectrum of clinical disease is different from that seen in district and provincial level hospitals due to referral of certain syndromes, more severe and non-responsive cases.

Secondly, during the study period, only a single blood culture was routine collected and as a cost saving technique, routine laboratory practice was that cultures showing obvious contamination (multiple organisms, skin flora etc.) were discarded before full work up without recording this finding. This will have lowered estimations of blood culture sensitivity, which can be improved with more blood cultures and higher volumes of blood [41, 42]. Additionally, although the isolates of Enterobacteriaceae members are almost always interpreted as true bloodstream infections, single blood culture practice may underestimate the roles of fastidious pathogens in the era of increased catheters and prosthetic devices-related bloodstream infections [43].

In our study hospital acquired BSI was defined as cases with positive blood culture after any hospitalization for more than $48 \mathrm{~h}$. Thus, patients who in reality had CABSI but with persistent bacteraemia, who were transferred after an inpatient stay at another hospital may have been misclassified as HABSI. The significance and role of certain bacteria, such as S. maltophilia or Aeromonas species, which have been associated with both pseudobacteraemia and real infection was not easy to establish because of a lack of repeat sampling in most of patients and the retrospective nature of the study. Lastly, clinical outcome analysis was only applied for $64.6 \%$ (477/738) of patients during the study period because the remaining patient's notes could not be retrieved. This has resulted in significant gaps in the data and may have introduced bias if the sample was not random.

\section{Conclusions}

Gram-negative bacteria are the most common cause of both community and hospital acquired blood stream infection in adults presenting to our tertiary referral hospital, with high associated mortality. The extended spectrum beta-lactamase (ESBL)-producing Enterobacteriaceae are prevalent in both community and health care setting. Our findings further support the need, as described in the GARP situation analysis [44] and the 2013 Vietnamese National Action Plan for AMR [45], for establishment of a comprehensive microbiological and antibiotic susceptibility surveillance system in Vietnam, with representation of the different levels of healthcare and to inform treatment guidelines and other public health measures.

\section{Abbreviations}

BSIs: Bloodstream infections; CABSI: Community-acquired bloodstream infections; ESBL: Extended Spectrum Beta-Lactamases; HABSI: Hospitalacquired bloodstream infections; IQR: Interquartile range; LMIC: Low and middle income countries; MICs: Minimum Inhibitory Concentrations; MRSA: Methicillin-resistant Staphylococcus aureus; NHTD: National Hospital for Tropical Diseases 


\section{Acknowledgements}

We gratefully acknowledge the bacteriology laboratory and General Planning Department in National Hospital for Tropical Diseases for providing the list of bacterial isolates, susceptibility results and for providing the list of medical records.

\section{Funding}

This study was funded by the Wellcome Trust Major Overseas Programme Vietnam

\section{Availability of data and materials}

The datasets used and/or analysed during the current study available from the corresponding author on reasonable request.

\section{Authors' contributions}

DVQ designed the study, collected \& analysed the data and wrote the first \& final draft; HNV, HNT, HTN, LBH, CLB collected, entered data, drafted sections of the manuscript; DVTV was responsible for cleaning data, analysis and drafted sections of the manuscript; KVN, TVN, DTT contributed to study design and were responsible for laboratory and clinical care, provided revisions of the manuscript edited the final draft; AT and HRVD helped with interpretation of the data, revision of the manuscript and edited the final draft; BN \& HFLW helped design the study, interpreted the data, revised the manuscript and edited the final draft. All authors approved of the final draft.

\section{Ethics approval and consent to participate}

Ethical approval was granted from the Ethics committee and review board of the National Hospital for Tropical Diseases, Hanoi, Vietnam (Approval No: 69/HDDD-NDTU). The NHTD ethics committee also approved the waiver of informed consent to participate in this study due to its retrospective design. All patient data were anonymised prior to the analysis.

\section{Consent for publication}

Not applicable.

\section{Competing interests}

HRVD is a member of the editorial board (Associate Editor) of the BMC Infectious Diseases. The authors otherwise declare that they have no competing interests.

\section{Publisher's Note}

Springer Nature remains neutral with regard to jurisdictional claims in published maps and institutional affiliations.

\section{Author details}

'Department of Infectious Diseases, Hanoi Medical University, Hanoi, Vietnam. ${ }^{2}$ National Hospital for Tropical Diseases, Hanoi, Vietnam. ${ }^{3}$ Wellcome Trust Major Overseas Programme, Oxford University Clinical Research Unit, Hanoi, Vietnam. ${ }^{4}$ Nuffield Department of Clinical Medicine, Centre for Tropical Medicine, University of Oxford, Oxford, UK. ${ }^{5}$ Department of Medical Microbiology, Radboudumc, Nijmegen, Netherlands.

Received: 15 March 2017 Accepted: 30 June 2017

Published online: 12 July 2017

\section{References}

1. Mortality GBD, Causes of Death C. Global, regional, and national age-sex specific all-cause and cause-specific mortality for 240 causes of death, 1990-2013: a systematic analysis for the Global Burden of Disease Study 2013. Lancet. 2015;385:117-71.

2. Goto M, Al-Hasan MN. Overall burden of bloodstream infection and nosocomial bloodstream infection in North America and Europe. Clin Microbiol Infect. 2013;19:501-9.

3. Laupland KB, Davies HD, Church DL, Louie TJ, Dool JS, Zygun DA, Doig CJ. Bloodstream infection-associated sepsis and septic shock in critically ill adults: a population-based study. Infection. 2004;32:59-64.

4. Nielsen SL. The incidence and prognosis of patients with bacteremia. Dan Med J. 2015;62

5. Southeast Asia Infectious Disease Clinical Research Network. Causes and outcomes of sepsis in Southeast Asia: a multinational multicentre crosssectional study. Lancet Glob Health. 2017;5:e157-67.
6. Melamed A, Sorvillo FJ. The burden of sepsis-associated mortality in the United States from 1999 to 2005: an analysis of multiple-cause-of-death data. Crit Care. 2009;13:R28.

7. Sogaard M, Norgaard M, Dethlefsen C, Schonheyder HC. Temporal changes in the incidence and 30-day mortality associated with bacteremia in hospitalized patients from 1992 through 2006: a population-based cohort study. Clin Infect Dis. 2011;52:61-9.

8. Mayr FB, Yende S, Angus DC. Epidemiology of severe sepsis. Virulence. 2014;5:4-11.

9. Cohen ML. Changing patterns of infectious disease. Nature. 2000;406:762-7.

10. Wertheim HF, Nghia HD, Taylor W, Schultsz C. Streptococcus suis: an emerging human pathogen. Clin Infect Dis. 2009;48:617-25.

11. Kiratisin P, Chongthaleong A, Tan TY, Lagamayo E, Roberts S, Garcia J, Davies T. Comparative in vitro activity of carbapenems against major Gramnegative pathogens: results of Asia-Pacific surveillance from the COMPACT ॥ study. Int J Antimicrob Agents. 2012;39:311-6.

12. Song JH, Jung SI, Ko KS, Kim NY, Son JS, Chang HH, Ki HK, Oh WS, Suh JY, Peck KR, et al. High prevalence of antimicrobial resistance among clinical Streptococcus pneumoniae isolates in Asia (an ANSORP study). Antimicrob Agents Chemother. 2004;48:2101-7.

13. Ferrer R, Martin-Loeches I, Phillips G, Osborn TM, Townsend S, Dellinger RP, Artigas A, Schorr C, Levy MM. Empiric antibiotic treatment reduces mortality in severe sepsis and septic shock from the first hour: results from a guideline-based performance improvement program. Crit Care Med. 2014;42:1749-55.

14. Paul M, Shani V, Muchtar E, Kariv G, Robenshtok E, Leibovici L. Systematic review and meta-analysis of the efficacy of appropriate empiric antibiotic therapy for sepsis. Antimicrob Agents Chemother. 2010;54:4851-63.

15. Vlieghe ER, Phe T, De Smet B, Veng HC, Kham C, Lim K, Koole O, Lynen L, Peetermans WE, Jacobs JA. Bloodstream infection among adults in Phnom Penh, Cambodia: key pathogens and resistance patterns. PLoS One. 2013;8:e59775.

16. Siegman-Igra Y, Fourer B, Orni-Wasserlauf R, Golan Y, Noy A, Schwartz D, Giladi M. Reappraisal of community-acquired bacteremia: a proposal of a new classification for the spectrum of acquisition of bacteremia. Clin Infect Dis. 2002;34:1431-9.

17. Kanoksil M, Jatapai A, Peacock SJ, Limmathurotsakul D. Epidemiology, microbiology and mortality associated with community-acquired bacteremia in northeast Thailand: a multicenter surveillance study. PLoS One. 2013;8:e54714.

18. Huong VTL, Ha N, Huy NT, Horby P, Nghia HDT, Thiem VD, Zhu X, Hoa NT, Hien TT, Zamora J, et al. Epidemiology, Clinical Manifestations, and Outcomes of Streptococcus suis Infection in Humans. Emerg Infect Dis. 2014;20:1105-14.

19. Cheng AC, Currie BJ. Melioidosis: epidemiology, pathophysiology, and management. Clin Microbiol Rev. 2005;18:383-416.

20. Clinical and Laboratory Standards Institute. Performance Standards for Antimicrobial Susceptibility Testing; Twenty-Third Informational Supplement. CLSI document M100-S23. Wayne: Clinical and Laboratory Standards Institute; 2013

21. Hoa NT, Chieu TT, Nghia HD, Mai NT, Anh PH, Wolbers M, Baker S, Campbell II, Chau NV, Hien TT, et al. The antimicrobial resistance patterns and associated determinants in Streptococcus suis isolated from humans in southern Vietnam, 1997-2008. BMC Infect Dis. 2011;11:6.

22. Nga TV, Parry CM, Le T, Lan NP, Diep TS, Campbell Jl, Hoang NV, Dung le T, Wain J, Dolecek C, et al. The decline of typhoid and the rise of non-typhoid salmonellae and fungal infections in a changing HIV landscape: bloodstream infection trends over 15 years in southern Vietnam. Trans $\mathrm{R}$ Soc Trop Med Hyg. 2012;106:26-34.

23. Nguyen KV, Thi Do NT, Chandna A, Nguyen TV, Pham CV, Doan PM, Nguyen AQ, Thi Nguyen CK, Larsson M, Escalante S, et al. Antibiotic use and resistance in emerging economies: a situation analysis for Viet Nam. BMC Public Health. 2013;13:1158.

24. Dao TT, Liebenthal D, Tran TK, Ngoc Thi Vu B, Ngoc Thi Nguyen D, Thi Tran HK, Thi Nguyen CK, Thi Vu HL, Fox A, Horby P, et al. Klebsiella pneumoniae oropharyngeal carriage in rural and urban Vietnam and the effect of alcohol consumption. PLoS One. 2014;9:e91999.

25. Moon HW, Ko YJ, Park S, Hur M, Yun YM. Analysis of community- and hospital-acquired bacteraemia during a recent 5-year period. J Med Microbiol. 2014;63:421-6.

26. Nakayama T, Ueda S, Huong BT, Tuyen le D, Komalamisra C, Kusolsuk T, Hirai I, Yamamoto Y. Wide dissemination of extended-spectrum beta- 
lactamase-producing Escherichia coli in community residents in the Indochinese peninsula. Infect Drug Resist. 2015;8:1-5.

27. Nguyen VT, Carrique-Mas JJ, Ngo TH, Ho HM, Ha TT, Campbell Jl, Nguyen TN, Hoang NN, Pham VM, Wagenaar JA, et al. Prevalence and risk factors for carriage of antimicrobial-resistant Escherichia coli on household and smallscale chicken farms in the Mekong Delta of Vietnam. J Antimicrob Chemother. 2015;70:2144-52.

28. Deen J, von Seidlein L, Andersen F, Elle N, White NJ, Lubell Y. Communityacquired bacterial bloodstream infections in developing countries in south and southeast Asia: a systematic review. Lancet Infect Dis. 2012;12:480-7.

29. Reddy EA, Shaw AV, Crump JA. Community-acquired bloodstream infections in Africa: a systematic review and meta-analysis. Lancet Infect Dis. 2010;10:417-32.

30. Skogberg K, Lyytikainen O, Ollgren J, Nuorti JP, Ruutu P. Population-based burden of bloodstream infections in Finland. Clin Microbiol Infect. 2012;18; E170-6.

31. Abernethy JK, Johnson AP, Guy R, Hinton N, Sheridan EA, Hope RJ. Thirty day all-cause mortality in patients with Escherichia coli bacteraemia in England. Clin Microbiol Infect. 2015;21:251.e251-8.

32. Anderson DJ, Moehring RW, Sloane R, Schmader KE, Weber DJ, Fowler VG $\mathrm{Jr}$, Smathers E, Sexton DJ. Bloodstream infections in community hospitals in the 21st century: a multicenter cohort study. PLoS One. 2014;9:e91713.

33. Van Nguyen K, Zhang T, Thi Vu BN, Dao TT, Tran TK, Thi Nguyen DN, Thi Tran HK, Thi Nguyen CK, Fox A, Horby P, Wertheim H. Staphylococcus aureus nasopharyngeal carriage in rural and urban northern Vietnam. Trans $R$ Soc Trop Med Hyg. 2014;108:783-90.

34. Thwaites GE. The management of Staphylococcus aureus bacteremia in the United Kingdom and Vietnam: a multi-centre evaluation. PLoS One. 2010;5:e14170

35. Allegranzi B, Bagheri Nejad S, Combescure C, Graafmans W, Attar H, Donaldson L, Pittet D. Burden of endemic health-care-associated infection in developing countries: systematic review and meta-analysis. Lancet. 2011:377:228-41.

36. Hongsuwan M, Srisamang P, Kanoksil M, Luangasanatip N, Jatapai A, Day NP, Peacock SJ, Cooper BS, Limmathurotsakul D. Increasing incidence of hospitalacquired and healthcare-associated bacteremia in northeast Thailand: a multicenter surveillance study. PLoS One. 2014:9:e109324.

37. Walton M, Harrison R, Chevalier A, Esguerra E, Van Duong D, Chinh ND, Giang H. Improving hospital death certification in Viet Nam: results of a pilot study implementing an adapted WHO hospital death report form in two national hospitals. BMJ Global Health. 2016;1

38. Falagas ME, Kastoris AC, Vouloumanou EK, Dimopoulos G. Communityacquired Stenotrophomonas maltophilia infections: a systematic review. Eur J Clin Microbiol Infect Dis. 2009:28:719-30.

39. Chang YT, Lin CY, Lu PL, Lai CC, Chen TC, Chen CY, Wu DC, Wang TP, Lin CM, Lin WR, Chen YH. Stenotrophomonas maltophilia bloodstream infection: comparison between community-onset and hospital-acquired infections. J Microbiol Immunol Infect. 2014;47:28-35.

40. Siebor E, Llanes C, Lafon I, Ogier-Desserrey A, Duez JM, Pechinot A, Caillot D, Grandjean M, Sixt N, Neuwirth C. Presumed pseudobacteremia outbreak resulting from contamination of proportional disinfectant dispenser. Eur J Clin Microbiol Infect Dis. 2007;26:195-8.

41. Lee A, Mirrett S, Reller LB, Weinstein MP. Detection of bloodstream infections in adults: how many blood cultures are needed? J Clin Microbiol. 2007:45:3546-8

42. Lin HH, Liu YF, Tien N, Ho CM, Hsu LN, Lu JJ. Evaluation of the blood volume effect on the diagnosis of bacteremia in automated blood culture systems. J Microbiol Immunol Infect. 2013;46:48-52.

43. Weinstein MP. Blood culture contamination: persisting problems and partial progress. J Clin Microbiol. 2003;41:2275-8.

44. The GARP Vietnam National Working Group. Situation Analysis Reports: Antibiotic use and resistance in Vietnam. Washington (District of Columbia): Center for Disease Dynamics, Economics and Policy; 2010.

45. Liew Kong Cheng. State of Play of Antimicrobial Resistance Research and Surveillance in Southeast Asia. SEA-EU-NET II. 2016.

\section{Submit your next manuscript to BioMed Central and we will help you at every step:}

- We accept pre-submission inquiries

- Our selector tool helps you to find the most relevant journal

- We provide round the clock customer support

- Convenient online submission

- Thorough peer review

- Inclusion in PubMed and all major indexing services

- Maximum visibility for your research

Submit your manuscript at www.biomedcentral.com/submit
Biomed Central 